\title{
Separating growth from regrowth
}

Neuronal remodelling, in which synapses and axons are pruned and may regrow to form new neuronal connections, is a feature of neural development. The molecular signalling underlying axon regrowth has been unclear. Now, however, a study in Drosophila melanogaster shows that the mechanism responsible for this regrowth involves the nuclear receptor UNF and is distinct to that underlying initial axon growth.

To explore axon regrowth during development, the authors studied the remodelling of mushroom body $\gamma$-neurons that occurs during metamorphosis in D. melanogaster. A genetic screening analysis, which involved the generation of mosaic flies, revealed that adult $\gamma$-neurons lacking UNF expression showed abnormal innervation of the mushroom body's $\gamma$-lobe, indicating that $\mathrm{UNF}$ is required for normal $\gamma$-neuron development.

In mutant unf $\gamma$-neurons in mosaic animals, axon development before axon pruning and the pruning process itself were normal. However, unlike wild-type axons, unf mutant axons showed no sprouting following pruning. These results indicate that axon regrowth after pruning but not initial axon growth requires UNF.

Mammalian target of rapamycin (mTOR) is implicated in promoting axon regrowth after injury, and a genomic screen in mice had suggested that the murine orthologue of UNF could downregulate the transcription of tuberous sclerosis 1 (Tsc1), which encodes a protein that inhibits mTOR. Thus, the authors hypothesized that UNF promotes $\gamma$-neuron axon regrowth during development through a mechanism involving repression of $T s c 1$ transcription and disinhibition of TOR.

In support of this hypothesis, mutation of Tor caused similar $\gamma$-neuron innervation defects to unf mutations, and TOR was not required for initial axon outgrowth. Furthermore, mutation of $T s c 1$ or

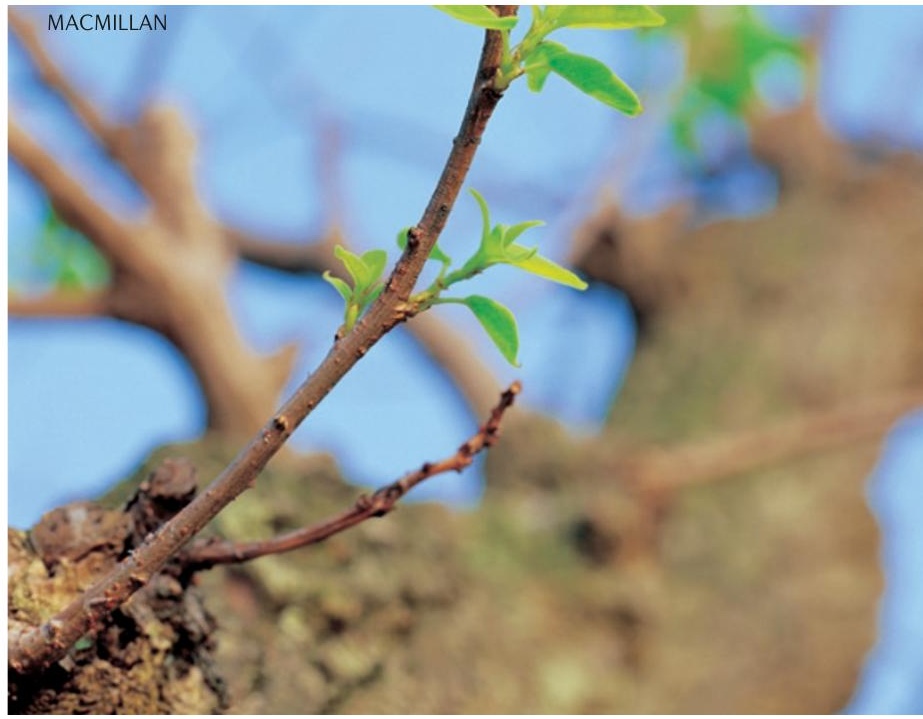

overexpression of components of the TOR signalling pathway in mutant unf $\gamma$-neuron clones partially rescued the abnormal $\gamma$-neuron phenotype.

Together, these results suggest that UNF exerts it effects on axon regrowth in part via TOR signalling, and that initial axon growth and axon regrowth following pruning involve different molecular mechanisms. The involvement of TOR in axon regrowth suggests that this process may share an underlying mechanism with axon growth following injury.

Darran Yates

ORIGINAL RESEARCH PAPER Yaniv, S. P. et al.

Axon regrowth during development and regeneration following injury share molecular mechanisms. Curr. Biol. 21 Aug 2012 (doi:10.1016/j.cub.2012.07.044) unlike wild-

type axons, unf mutant

axons showed

no sprouting

following

pruning 